\title{
Cultura tributaria: lineamientos para la comnensación de saldos a favor de los contribuyentes
}

\author{
Tax culture: Guidelines for the \\ compensation of balances in favor of \\ taxpayers \\ Cultura tributária: Diretrizes para a \\ compensação de saldos a favor do \\ contribuinte
}

\section{Luz Mary Barros Bayona ${ }^{1}$}

\section{René Segundo Hernández Igirio² \\ Víctor Hugo Martínez González ${ }^{3}$ \\ Carlos Andrés Ochoa Triana ${ }^{4}$ \\ Universidad Popular del Cesar}

1 Profesor de la Universidad Popular del Cesar Valledupar, Colombia. Contador Público luzbarros@unicesar.edu.co https://orcid.org/0000-0002-1900-5640

2 Rene Segundo Hernández Igirio MSc. En Gerencia de Recursos Humanos. Contador Público renehernandez@unicesar.edu.c

Universidad Popular del Cesar

https://orcid.org/0000-0002-7998-1694

Doctor en Ciencias Gerenciales

3 Profesor de la Universidad Popular del Cesar Valledupar, Colombia. Contador Público https://orcid.org/0000-0002-0727-0391 victormartinez@unicesar.edu.co

\section{RESUMEN}

En el sector tributario colombiano se contempla la verificación de las retenciones o pago en exceso que permite generar saldo a favor del contribuyente, lo que se considera relevante para la creación de una cultura tributaria que permita educar a la ciudadanía ante el comportamiento de sus aportes fiscales obligatorios, dando un interesante giro para evitar la evasión de impuestos, pues al conocer de forma transparente hacia dónde va el dinero y 
cómo lo ocupa el Estado en beneficios sociales, se ve retribuido a manera de inversión social. Este trabajo refleja la necesidad imperativa de presentar la importancia de la cultura tributaria y dar a conocer lineamientos para la compensación de saldos a favor del contribuyente.

\section{PALABRAS CLAVE:}

Cultura tributaria, Lineamientos, Saldo a favor, contribuyentes.

\section{ABSTRACT}

In the Colombian tax sector, the verification of withholdings or overpayment is contemplated, which allows generating a balance in favor of the taxpayer, which is considered relevant for the creation of a tax culture that allows educating citizens about the behavior of their contributions. Mandatory tax authorities, giving an interesting twist to avoid tax evasion, because by knowing in a transparent way where the money is going and how the State uses it in social benefits, it is rewarded as a social investment. This work reflects the imperative need to present the importance of the tax culture and present guidelines for the compensation of balances in favor of the taxpayer.

\section{KEYWORDS:}

Tax culture, Guidelines, Balances in favor, Taxpayers

\section{RESUMO}

No setor tributário colombiano, contemplase a verificação de retenções ou pagamentos indevidos, o que permite gerar um equilíbrio a favor do contribuinte, o que é considerado relevante para a criação de uma cultura tributária que permita educar o público sobre o comportamento dos Autoridades fiscais obrigatórias, o que dá um toque interessante para evitar a evasão fiscal, porque ao saber de forma transparente para onde vai o dinheiro e como o Estado o utiliza nas prestações sociais, é recompensado como investimento social. Este trabalho reflete a necessidade imperiosa de apresentar a importância da cultura tributária e apresentar diretrizes para a compensação de saldos a favor do contribuinte.

\section{PALAVRAS-CHAVE:}

Cultura tributária, Diretrizes, Saldos a favor, Contribuintes

\section{INTRODUCCIÓN}

En el ámbito financiero, la declaración de impuestos resulta ser el canal que tiene el Estado para obtener ingresos de los contribuyentes que le permitan satisfacer las necesidades de la comunidad que gerencia, de este modo, los ciudadanos pueden percibir que estos tributos se utilizan en pro de su bienestar, originando una mejor calidad de vida y un crecimiento organizado de la sociedad a la que pertenece.

En este sentido, la cultura tributaria representa una herramienta imprescindible para la población, dado que proporciona comprensión y transparencia ante el comportamiento de sus tributos en manos del Estado. De igual manera ejerce como una poderosa fuente de información aportando datos que estimulen el cumplimiento de esta obligación y de este modo reduciendo significativamente la evasión de impuestos.

Resulta importante destacar un factor poco conocido como lo es el proceso de devolución de saldos a favor mediante la declaración de impuestos de renta a los usuarios que cumplan con los requisitos establecidos. De esta manera, en este artículo se pretende informar los elementos necesarios para obtener esta compensación ante la División de Recaudo y Devolución de la Dirección de Impuestos y Aduanas Nacionales, en adelante, (DIAN), por lo cual la cultura tributaria tiene un rol preponderante para atraer la mirada de los contribuyentes en materia de recaudación, 
debido a que también les permite tener criterios, hábitos y actitudes con respecto a la tributación.

\section{LA CULTURA TRIBUTARIA, PODEROSA HERRAMIENTA DE INFORMACIÓN.}

Resulta interesante medir el impacto que tiene la cultura tributaria ante el logro de las metas del ente recaudador y el comportamiento del contribuyente, pues la disminución del aporte voluntario no es un indicador del alcance de la cultura tributaria. La recaudación depende en gran medida de la campaña informativa que haga el Estado y de la retribución que la población perciba en el uso de sus tributos. En este sentido, la buena gestión por parte de las instituciones se considera imprescindible para la base de una cultura tributaria que permita observar los datos de manera transparente y esto a su vez permite incentivar el pago de los impuestos bajo la sensación de un uso eficiente de los recursos públicos.

Considerada como "el conjunto de acciones con pretensión de sistematicidad que se financian mediante presupuesto de inversión y se diseñan con objetivos directos e impactos indirectos con el fin de mejorar la disposición de un sistema político a aceptar voluntariamente la carga y procedimientos de tributos, tasas y contribuciones que se destinan a la provisión de bienes y servicios comunes o bienes y servicios meritorios para el mismo sistema político que decide imponérselas", según Bromberg (2009) la cultura tributaria, establece una clara y fuerte conexión informativa que proporciona armonía en un camino bidireccional donde el usuario encuentra respuestas a sus dudas sobre los intrincados procesos tributarios.

Es interesante resaltar, que el comportamiento en el recaudo es el resultado de la percepción que el ciudadano mantiene frente a las obligaciones tributarias, lo que demuestra que existe una cultura tributaria en Colombia, sin embargo, ésta debe fortalecerse, mediante la transmisión constante de información veraz y transparente al público que cumple con su deber de pago de obligaciones fiscales para luego exigir sus derechos sociales.

Por su parte, Rodríguez (2011) indica que "resulta un problema social que consiste en conseguir que la ciudadanía se convenza de la posibilidad, la necesidad y la conveniencia de una fiscalidad justa", esto incide en que la aceptación social influye en la integridad como ciudadano, de manera que el cumplimiento oportuno de sus tareas, al facturar, archivar y preparar sus rendiciones lo hacen representante de una imagen social exitosa.

De igual manera, este llamado problema social, tendría otro matiz, si desde la escuela los niños y jóvenes tuviesen la adecuada educación fiscal, que los prepare para tener conciencia social como contribuyente, fomentando su compromiso con la comunidad y desarrollando su sentido de pertenencia, transmitiendo así valores, actitudes y responsabilidad tributaria, lo que evitaría masivamente conductas fraudulentas y evasoras de la obligación fiscal.

Cabe destacar que resulta importante estudiar al contribuyente, pues es a través de éste, que "se materializa el buen funcionamiento de la política fiscal y económica mediante el pago o no pago de sus obligaciones fiscales", según señala Velásquez (2009). En este mismo orden de ideas, el colombiano promedio, considera que el pago de impuestos representa un deber moral como ciudadano y a su vez un deber social pensando como ser gregario en la garantía de los servicios públicos que benefician a la comunidad donde pertenece.

\section{COMPENSACIÓN O DEVOLUCIÓN}

Esta figura tributaria, hace referencia al cruzar un saldo a favor del contribuyente con una deuda que tiene ante la DIAN. Mientras que la devolución, permite el reintegro del saldo a favor 
mediante títulos de Devolución de Impuestos, y esto ocurre cuando el valor de la devolución supere las 1.000 UVT, de modo que en caso de que el valor del saldo a favor sea igual o inferior a 1000 UVT, se hará un abono a la cuenta bancaria del solicitante.

Así lo explican Briceño y Vergara (2002) cuando indican que "la figura por la cual dos personas son deudoras una de la otra al mismo tiempo, para que la compensación pueda obrar debe tratarse de obligaciones que tengan un objeto o una prestación de la misma naturaleza. En materia fiscal, solo es admisible cuando lo autorice expresamente una ley".

En el caso colombiano, la compensación se solicita cuando la declaración del IVA resulta con saldo a favor y además existe una obligación tributaria pendiente con la DIAN a razón de retenciones, impuestos o anticipos.

La DIAN 014017 (2009), por su parte, señala que de acuerdo al artículo 15 del Estatuto Tributario, la compensación podrá ser parcial, donde no necesariamente los saldos de acreedor tienen que ser exactamente iguales y el remanente puede extinguirse de otra forma, con el pago o solicitar su devolución, para lo cual ciertas condiciones aplican.

La devolución consiste entonces según la visión de Vasco (2009) en "el reintegro de las sumas a favor de los contribuyentes o responsables de las personas que han pagado sumas que no debían o pagos en exceso".

En el caso de solicitud de devoluciones así como de las compensaciones de saldo a favor, éstas serán presentadas a más tardar dentro de los dos años siguientes al vencimiento de termino de la declaración. $Y$ esto representa un proceso que exige la presentación de requisitos estipulados en la normativa vigente de Ley.

\section{SALDO A FAVOR, UNA EXPERIENCIA TRIBUTARIA}

En materia tributaria, los saldos a favor tienen su origen en retenciones en la fuente, auto retenciones liquidadas, anticipos, beneficios tributarios o arrastres de saldos a favor de años anteriores. Colombia en este rubro considera tres normas que fundamentan el procedimiento para que las personas naturales o jurídicas puedan solicitar un saldo a favor ante las Autoridades del fisco nacional, a saber: a) Orden Administrativa 0004 del 30 de abril de 2002. b) Decreto 1000 del 8 de abril de 1997, y c) Artículos del 850 al 865 del Estatuto Tributario Nacional, donde se indica la aplicación de dichas normativas garantizando los mejores y más eficientes procesos de fiscalización por parte de las Autoridades Fiscales ante los contribuyentes.

En este sentido, resulta interesante destacar que la solicitud de un saldo a favor constituye transferir recursos por parte del Estado hacia los contribuyentes, por lo cual se considera importante conocer los procedimientos precisos para dicha recuperación monetaria.

Según el estatuto Tributario, Artículos 815 y 850 , los usos que un contribuyente o usuario aduanero puede darle a un saldo a favor son:

- $\quad$ Solicitud en compensación

- $\quad$ Solicitud en devolución

- Imputación en la liquidación privada de un impuesto.

Aclaradas las anteriores definiciones de compensación y devolución, se especifica que, una imputación es la acción por medio de la cual el contribuyente y responsable, decide trasladar a su declaración tributaria del periodo actual, el saldo a favor determinado en la liquidación privada de sus declaraciones tributarias o de una actuación oficial mediante los procedimientos y 
presentación de requisitos establecidos para dicho fin.

También puede suceder que el saldo a favor se origine en el Impuesto sobre las ventas y en este caso son susceptibles de devolución según los parágrafos $1^{\circ}$ y $2^{\circ}$ del Artículo 850 del Estatuto Tributario en dos rubros

1 Responsables del impuesto sobre las ventas:

a. Responsables de bienes y servicios contemplados en el Art. 481 del Estatuto Tributario.

b. Productores de los bienes exentos señalados en el art. 477 del Estatuto Tributario.

c. Responsables de los bienes y servicios incluidos en los art. 468-1 y 468-3 del Estatuto Tributario

d. Aquellos que hayan sido objeto de retención.

2. Aquellos que hayan pagado en la adquisición de materiales para la construcción de vivienda de interés social y prioritario.

\section{¿PUEDE VENCERSE EL LÍMITE DE TIEMPO PARA UN SALDO A FAVOR?}

La devolución de saldos a favor de impuestos tiene una fecha límite que deberá presentarse a mas tardar dos años después de la fecha de vencimiento del término para hacer la declaración.

Así lo establece el Estatuto Tributario en su Art. 854, explicando que cuando dicho saldo a favor de la declaración de impuesto haya sido modificado mediante una liquidación oficial sin efectuar la devolución la parte rechazada, no podrá ser solicitada aunque esta liquidación haya sido impugnada, hasta que se resuelva definitivamente la procedencia del saldo.
Así mismo, es necesario conocer que la DIAN cuenta con cincuenta (50) días para dar respuesta ante la solicitud y podrá suspenderse hasta por noventa (90) días si la División de Fiscalización requiere investigar algún asunto de la declaración tributaria que es objeto de dicha devolución, esto reza en el Artículo 855 del Estatuto Tributario.

\section{INADMISIONES DEL SALDO A FAVOR}

Existen causales por las cuales la DIAN puede rechazar la solicitud de devolución, y esta puede ser parcial o total según los siguientes casos según lo establecido en el decreto 2627 de 1993, artículo 7:

- $\quad$ El periodo solicitado ya ha sido objeto de solicitud de devolución,

- El solicitante no haya cancelado el impuesto a las ventas del que ha sido objeto dicha solicitud.

- $\quad$ Cuando no se acredite el cumplimiento de los requisitos señalados en el auto de inadmisión

- $\quad$ Cuando no se interponga el recurso de reposición se hace de forma extemporánea

- El solicitante no tiene derecho a devolución

- La providencia que rechaza la solicitud de devolución debe proferirse dentro de los treinta (30) días siguientes a la fecha de presentación de la solicitud.

De igual manera se considera improcedente la solicitud de devolución o compensación cuando la Administración constate que no ha sido efectuado el impuesto sobre ventas por parte del solicitante, Cuando el impuesto sobre las ventas haya sido solicitado como descontable de la cuenta de impuesto a las ventas por 
pagar o cuando no se cumple con los requisitos en la contabilidad. Así mismo, también será derogado cuando se obtenga la devolución o compensación utilizando documentos falsos o mediante fraude, lo que equivale una sanción del $500 \%$ de la suma compensada, esto está contemplado en el decreto 1243 de 2001, Artículo 11

\section{CIFRAS ALARMANTES DE LOS PRINCIPALES MOTIVOS POR LOS CUALES NO SE SOLICITA DEVOLUCIÓN O COMPENSACIÓN}

Según Revelo (2012) en el XVII Congreso Internacional de Contaduría, Administración e Informática en la Ciudad de México, se expusieron los motivos por los cuales no se solicita la devolución o compensación de saldo a favor en el impuesto sobre las ventas en Colombia y las principales se mencionan a continuación:

\section{Temor a la fiscalización de la DIAN}

Se ha demostrado que existe una relación muy estrecha entre temor y evasión, de modo que, una adecuada cultura tributaria, tendría un efecto totalmente opuesto, generando información oportuna que disipe las dudas de los contribuyentes y minimizando el comportamiento evasor.

Se encienden las alarmas ante una inspección de la DIAN, pues al no tener esta cultura tributaria, los incumplimientos en materia de requisitos, recaudos, normativa y obligaciones se hacen densos para la comprensión. En este sentido, el porcentaje de contribuyentes que le temen a una fiscalización raya en más del $95 \%$ y según Bernal (2003), este comportamiento obedece a la carencia de conciencia tributaria, de igual modo a un sistema tributario poco transparente, que exige mediante decretos, reglamentos e imposiciones, pero no retribuye al menos con información al contribuyente.
En este mismo sentido, la inflexibilidad de la administración tributaria plantea distanciamiento natural y finalmente desencadena en comportamiento de evasión fiscal. Esta característica se puede reducir significativamente, al disminuir las brechas entre la obligación y el cumplimiento y eso solo se logra con cultura tributaria.

\section{Desconocimiento}

Los productores consideran que son escasas las orientaciones sobre el tema de Administración tributaria que están al alcance del contribuyente, por lo cual un alarmante $85 \%$ de este rubro alude no realizar la devolución /compensación dada la falta de incentivos o por el hecho de no tener información suficiente en estos beneficios. Lo cual puede representar un costo extra al tener que contratar un asesor tributario que lo oriente de forma correcta, por consiguiente, prefieren obviar este recurso.

\section{Falta de tiempo para cumplir con los procesos}

Esta cifra se considera importante al tratarse de un $49 \%$ de contribuyentes que no realizan esta solicitud de saldo a favor pues para cumplir con los requisitos exigidos demanda tiempo considerable para elaborar los reportes correspondientes.

\section{Falta de interés}

En este congreso se determinó que un $5 \%$ de los productores consideran innecesario solicitar el beneficio de saldo a favor, La carencia de una verdadera cultura tributaria, aunado al desinterés de los productores, propicia un caldo de cultivo para que se refleje la apatía frente al sistema tributario colombiano lo que conlleva a la evasión fiscal. 


\section{CONCLUSIONES}

La relevancia que ha mostrado la cultura tributaria en brindar conocimiento a la sociedad y en especial a los contribuyentes se hace notoria. Existe una gran cantidad de leyes, resoluciones, decretos sobre procesos como el tratado en este trabajo, sobre la devolución y/o compensación, de manera que no se ofrece suficiente claridad dado el complejo sistema tributario que los origina, de este modo, resulta imperante impulsar estrategias que revelen datos precisos sobre el comportamiento de los tributos, pues, muy a pesar de las circunstancias sociales y políticas, los ciudadanos pagan impuestos, está demostrado que las metas de recaudación han aumentado considerablemente, Entonces se considera necesario un plan de lineamientos que permitan educar a la ciudadanía a mantener activo su pago oportuno lo que redunda en el bienestar social de la Nación, de igual manera el beneficio que se obtiene al conocer información sobre saldos a favor, entre otros derechos, garantiza la armoniosa relación entre las partes involucradas.

\section{REFERENCIAS BIBLIOGRÁFICAS}

Bernal, A. (2003). La penalización de la evasión fiscal dentro de un entorno de justicia tributaria. Trabajo de grado. Pontificia Universidad Javeriana. Facultad de Ciencias Jurídicas. Bogotá, Colombia.

Briceño, T., \& Vergara, R. (2002). Diccionario Técnico Tributario. Medellín: Centro Interamericano Jurídico Financiero.

Bromberg, P. (2009). Cultura tributaria como política pública. Contrato 629 de 2009. Instituto de Estudios Urbanos de la Universidad Nacional de Colombia.
Conceptos y oficios DIAN Dirección de Impuestos y Aduanas Nacionales

Decreto 1000 del 8 de abril de 1997

Decreto 1243 de 2001 Artículo 11

Decreto 2627 del año 1993 Artículo 7

Estatuto Tributario Nacional

Hernández, C. (2020) Compensación de saldos a favor a contribuyentes en medio de crisis sanitaria, disponible en: https://incp. org.co/compensacion-de-saldos-a-favor-acontribuyentes-en-medio-de-crisis-sanitarial consultado en marzo 2021

Orden Administrativa 0004 del 30 de abril de 2002

Revelo, L. (2012) La devolución y/o compensación de saldos a favor en el impuesto sobre las ventas. Reflexiones frente a la legislación colombiana. Ponencia ante el Congreso Internacional de Contaduría, administración en Informática. México

Rodríguez, J. (2011). Cultura tributaria. Propuestas y argumentos para aumentar la justicia fiscal. Paraguay.

Velásquez, T. (2009). "La sociología de las finanzas públicas". En: Imaginales No. 7

Vasco, R. (2009). Procedimiento tributario 2009. Bogotá: Centro Interamericano Jurídico Financiero. 